\title{
On Equational Theories, Unification and Decidability
}

\author{
Hans-Jürgen Bürckert, Alexander Herold and Manfred Schmidt-Schauß \\ FB Informatik, Universität Kaiserslautern, Postfach 3049, D-6750 Kaiserslautern, W.-Germany \\ net-address: UUCP; ... Imcvaxlunidoluklirb!buerkert
}

\begin{abstract}
:
The following classes of equational theories, which are important in unification theory, are presented: permutative, finite, Noetherian, simple, almost collapse free, collapse free, regular, and $\Omega$-free theories. The relationships between the particular theories are shown and the connection between these classes and the unification hierarchy is pointed out. We give an equational theory that always has a minimal set of unifiers for single equations, but there exists a system of two equations which has no minimal set of unifiers. This example suggests that the definition of the unification type of an equational theory has to be changed. Furthermore we study the conditions, under which minimal sets of unifiers always exist.

Decidability results about the membership of equational theories to the classes above are presented. It is proved that Noetherianness, simplicity, almost collapse freeness and $\Omega$-freeness are undecidable. We show that it is not possible to decide where a given equational theory resides in the unification hierarchy and where in the matching hierarchy.
\end{abstract}

\section{Introduction}

Unification theory is concerned with problems of the following kind: given two terms built from function symbols, constants and variables, do there exist terms that can be substituted for the variables such that the two terms thus obtained become equal? Robinson [Ro 65] was the first who gave an algorithm to find such a substitution with the additional property that this returned 'unifier' is most general (or is an mgu for short), i.e. all other substitutions 'unifying' the two terms can be computed from that substitution. From an algebraic point of view unification is solving equations and an mgu generates the whole set of solutions.

Equational unification extends the Robinson unification problem to solving equations in equationally defined theories. But then there may not exist a single mgu. Depending on the equational theory there are finite or infinite sets of mgu's and in some cases the set of mgu's does not even exist. The equational theories can therefore be classified into unitary, finitary and infinitary unifying theories and the class of nullary unifying theories. This classification is known as the unification hierarchy [Si 84, Si 86]. Sometimes one is not interested in full but only in one-sided unification. This opcration is called matching and there is an analogous matching hierarchy.

Solving single equations is a special case of solving systems of equations. If there is at least one binary free function symbol, both problems are equivalent in the sense that one can always construct a single equation with exactly the same solution behaviour as the equation system that has to be solved. However, as an example shows, the set of mgu's may always exist for single equations, while this need not be the case, if we consider equation systems.

Investigations in unification theory lead to a number of classes of equational theories that are not defined in terms of unification theory. These classes are often introduced to generalize results about special equational theories to a whole family of equational theories. For example regularity - equal terms have the same variables - and collapse freeness of an equational theory - non-variables are not equal (don't 'collapse') to variables - play a prominent role in the study of combining unification algorithms [He 86, Ki 85, Ti 86, Ye 85]. Certain collapsing terms do not disturb the unification behaviour; equational theories that have only collapsing terms of that kind, if any, are called almost collapse free [Bü 86]. The notion of collapse freeness can be generalized to theories without any subterm collapsing equations, 
i.e. theories where no term is equal to any proper subterm of its [He 86]. We call those theories simple since they admit the simple occurs-check as in the Robinson case. Theories that behave similar to the empty theory in that the converse of the congruence condition holds, are called $\Omega$-free: equal terms starting with the same function symbol have cqual immediate subterms [ $\mathrm{Sz} 82]$. Equational theories with finite equivalence classes have the important property that the set of most general unifiers always exists. The class of finite theories is a subclass of the Noetherian equational theories, where the subsumption relation is a well-founded (or Noetherian) quasi-ordering. Important examples for finite theories are the permutative theories where equal terms have the same symbols.

In the literature the terminology for the classes listed above is confusing, so sometimes theories with the same symbois on both sides are called permutative [La 77] and sometimes the superclass with finite equivalence classes is called permutative [ $\mathrm{Sz} \mathrm{82,} \mathrm{Ki} \mathrm{85].} \mathrm{Collapse} \mathrm{free} \mathrm{theories} \mathrm{are} \mathrm{called} \mathrm{confined} \mathrm{in} \mathrm{[Ye} \mathrm{85]} \mathrm{and} \mathrm{theories} \mathrm{with} \mathrm{collapse} \mathrm{equations}$ are said to be 'potent' (in French) [Ki 85]. Another problem is that some of the publications are not available for everybody, since there are only versions in German or in French. So one aim of this paper was to unify the definitions and terminology of the above classes and to collect the most important results about these theories known so far. On the other hand we investigated the relationships between these theories and we classify them in a hierarchy. We introduce a whole class of examples of equational theories, the monadic theories, which were useful in finding examples and counterexamples, especially when we studied how the above classes fit into the unification hierarchy.

The monadic theories were also useful in solving decidability problems. We investigated the decidablity of the class problem for the above theories, i.e. the problem whether an equational theory belongs to a certain class or not. We show that except for the trivially decidable cases - regularity, collapse freeness and permutativity - all other class problems are undecidable. Finally we demonstrate that it is neither possible to decide where equational theories reside in the unification hierarchy nor where in the matching hierarchy.

For space limitations we omitted all proofs. They are carried out in detail in a full version of this paper [BH 86]. However, for the most important proofs a sketch is given.

\section{Basic Definitions and Notations}

Unification theory rests upon the usual algebraic notions (see e.g. [Gr79, BS 81]) with the familiar concept of an algebra as a pair $(A, \mathbb{F})$ where $A$ is the carrier and $F$ is a family of operators given with their arities.

For $\mathbb{F}$ and a denumerable set of variables $V$, we define the set of first order terms $\mathbb{T}$ over $F$ and $V$, as the least set with (i) $V \subseteq \mathbb{T}$, (ii) if $t_{1}, \ldots, t_{n} \in \mathbb{T}$ and arity $(f)=n$ then the string $f\left(t_{1} \ldots t_{n}\right) \in \mathbb{T}$. For unary function symbols $f$ we use the abbreviation $\mathrm{f}^{\mathrm{n}}(\mathrm{x})=\mathrm{f}\left(\mathrm{f}^{\mathrm{n}-1}(\mathrm{x})\right)$ and $\mathrm{f}^{\mathrm{f}}(\mathrm{x})=\mathrm{x}$. Let $\mathrm{V}(\mathrm{s})$ be the set of variables occurring in a term $\mathrm{s}$; a term $\mathrm{s}$ is ground if $V(s)=\varnothing$. Let $\#(X, s)$ denote the number of occurrences of a symbol $X \in F \cup \mathbb{V}$ in a term $s$.

The algebra with carrier $\mathbb{T}$ and with operators corresponding to the term constructors of $\mathbb{F}$, is the absolutely free (term) algebra. If the carrier is the set of ground terms, it is the initial algebra [GT 78] or Herbrand Universe [Lo 78].

A substitution $\sigma: \mathbb{T} \rightarrow \mathbb{T}$ is an endomorphism on the term algebra which is identical almost everywhere on $\mathbb{V}$, and therefore can be represented as a finite set of variable-term pairs $\sigma=\left(x_{1} \leftarrow t_{1}, \ldots, x_{n} \leftarrow t_{n}\right)$. The restriction $\sigma_{v}$ of a substitution $\sigma$ to a set of variables $\mathrm{V}$ is defined as $\left.\sigma\right|_{\mathrm{V}} \mathrm{x}=\sigma \mathrm{x}$ if $\mathrm{x} \in \mathrm{V}$ and $\sigma \mathrm{l}_{\mathrm{V}} \mathrm{x}=\mathrm{x}$ else. $\bar{\Sigma}$ is the set of all substitutions and $\varepsilon$ denotes the identity. The application of a substitution $\sigma$ to a term $t \in T$ is written as $\sigma$. The composition of substitutions is defined as the usual composition of mappings $(\sigma \cdot \tau) t=\sigma(\tau t)$ for $t \in \mathbb{E}$ and usually denoted by $\sigma \tau$.

Let $\operatorname{DOM}(\sigma)=\{\mathrm{x} \in \mathrm{V} \mid \sigma \mathrm{x} \neq \mathrm{x}\}$ denote the domain of $\sigma, \operatorname{COD}(\sigma)=\{\sigma \mathrm{x} \mid \mathrm{x} \in \operatorname{DOM}(\sigma)\}$ the codomain of $\sigma$, and $\operatorname{VCOD}(\sigma)=\operatorname{V}(\operatorname{COD}(\sigma))$ the set of variables introduced by $\sigma$. If $\operatorname{VCOD}(\sigma)=\varnothing$ then $\sigma$ is a ground substitution. A substitution $\rho$ is called a renaming substitution, iff $\operatorname{COD}(\rho) \subseteq \mathbb{V}$ and $\rho x=\rho y$ implies $x=y$ for $x, y \in D O M \rho$. 
An equation is a pair of terms usually denoted by $s=t$. Given a set of equations $E$ and a single equation $s=t$ we write $\mathrm{E}=\mathrm{s}=\mathrm{t}$ to denote that $\mathrm{s}=\mathrm{t}$ is true in every model of $\mathrm{E}(\mathrm{s}=\mathrm{t}$ is a consequence of $\mathrm{E})$. An equational theory is a set of equations $T$ with $T=s=t$, iff $s=t$ is in $T$, i.e., $T$ consists exactly of all its consequences. The least equational theory $T(E)$ that contains a given set of equations $E$ is just the finest congruence on the term algebra that contains all pairs $\sigma s=\sigma t$, for all equations $s=\imath$ in $E$ and all substitutions $\sigma$ (the substitution invariant congruence generated by $E$ ). We say $s$ and $t$ are $E$-equal, abbreviated by $s=t$, iff the terms $s$ and $t$ are in this congruence. $E$ is a presentation of the congruence ${ }^{\prime}={ }_{\mathrm{E}}$ ' or an axiomatization of the equational theory $\mathrm{T}(\mathrm{E})$, and sometimes we speak - for short - of the 'theory $E$ ' to denote the equational theory axiomatized by $E$. Obviously the axiomatization for an equational theory is not unique. A theory that admits a finite axiomatization is called finitely generated, otherwise it is infinitely generated. An equational theory is consistent iff different variables are not equal in this theory, i.e., there are non-trivial models.

Birkhoff gave rules for the derivation of an equation $s=t$ from an axiomatization $E$ (abbreviated by $E r s=t$ ) that are equivalent to the generation rules of the congruence ' $=$ '. His well-known completeness theorem of equational logic [Bi 35] states the equivalence between the model theoretic and this proof theoretic notion:

Theorem 2.1: $\quad E=s=t$ iff $\quad E-s=t \quad$ (iff $\left.\quad s={ }_{E} t\right)$.

A term rewriting system is a set of directed equations or rules $R=\left(l_{1} \rightarrow r_{1}, \ldots, l_{n} \rightarrow r_{n}\right\}$ with $\vee\left(r_{i}\right) \subseteq V\left(l_{i}\right)$. A rewriting step from a term $t$ is an application of $R$ to $t$ in the following way: if there is a subterm $s$ of $t$ and a rule $l \rightarrow r$ and a substitution $\sigma$ with $s=\sigma l$ then replace $s$ in $t$ by $\sigma$. This defines a reduction relation on terms denoted by $\rightarrow$. We say a term $\mathrm{t}$ reduces to a term $\mathrm{b}$, denoted by $\mathrm{t}{ }_{\mathrm{*}} \mathrm{R} \mathrm{u}$ iff $\mathrm{u}$ can be reached from $\mathrm{t}$ by finitely many rewriting steps. A rewrite system is called canonical or complete, if it is both Noetherian (there are no infinite reduction sequences) and confluent (for two different reductions of a term $t$ to terms $p$ and $q$ there is a term $s$ that can be reached by reductions from both $p$ and $q$ ). Every canonical term rewriting system $R$ defines a unique normal form $\|s\|_{R}$ of a term $s$ and yields a decision procedure for the corresponding $E$-equality: $s={ }_{E} t$ iff $\|s\|_{R}=\|t\|_{R}$ (R has the Church-Rosser property). A terminating term rewriting system can sometimes be completed to a canonical system with the Knuth-Bendix completion procedure [KB 70]. Rewrite systems in the word monoid are also known as Semi-Thue systems. Our definitions and notations are consistent with [Gr 79, HO 80, Ta 79, Bu 85].

$E$-equality is extended to substitutions by $\sigma=_{\mathrm{B}} \tau[\mathrm{W}]$ iff $\sigma \mathrm{x} \mathrm{E}_{\mathrm{B}} \tau \mathrm{x}$ for all $\mathrm{x} \in \mathrm{W}(\sigma$ and $\tau$ are $E$-equal on $W$ ).

A term $t$ is more general than a term $s$ (or $s$ is an E-instance of t):

$$
s \geq_{E} \quad \text { iff } \quad \exists \lambda \in \Sigma: s={ }_{E} \lambda t
$$

and two terms $s, t$ are called E-equivalent $s=_{B} t$ iff $s \geq_{E} t$ and $t \geq_{E} s$.

These notions are extended to substitutions by:

$$
\begin{array}{llll}
\sigma \geq_{\mathrm{E}}:[W] & \text { iff } & \exists \lambda \in \mathbb{E}: \sigma=_{\mathrm{E}} \lambda \tau[\mathrm{W}] & (\sigma \text { is an E-instance of } \tau \text { on } W) \\
\sigma \bar{\Sigma}_{\mathrm{E}} \tau[\mathrm{W}] & \text { iff } & \sigma \geq_{\mathrm{E}} \tau[\mathrm{W}] \text { and } \tau \geq_{\mathrm{E}} \sigma[\mathrm{W}] & (\sigma, \tau \text { are E-equivalent on } W)
\end{array}
$$

We use $>$ for the strict E-instance relation.

A finite system of equations $\left.\Gamma:=\left[s_{i}=t_{j} ; 1 \leq i \leq n\right)\right\}$ together with a theory $E$ is called an E-unification problem or an equation system and it is often denoted as $\left\langle\mathrm{s}_{\mathrm{i}}=\mathrm{t}_{\mathrm{i}}: 1 \leq \mathrm{i} \leq \mathrm{n}\right\rangle_{\mathrm{E}}$. A substitution $\sigma$ is an $E$-unifier or a solution of $\Gamma$, iff $\sigma s_{i}={ }_{E} \sigma t_{i}(1 \leq i \leq n)$. The set of all E-unifiers of $\Gamma$ is denoted as $U_{E}(\Gamma)=U_{E}\left(s_{i}=t_{i}: 1 \leq i \leq n\right)$. Without loss of generality the unifiers of $\Gamma$ are idempotent (if not, one can always find equivalent unifiers that are idempotent). For a given E-unification problem $\Gamma$, it is not necessary to compute the whole set of unifiers $U_{E}(\Gamma)$ - which is always recursively enumerable - , but instead smaller sets representing $\mathrm{U}_{\mathrm{E}}(\Gamma)$. Therefore we define a complete set of E-unifiers of $\Gamma, \mathrm{cU}_{\mathrm{E}}(\Gamma)$, on $\mathrm{W}=\mathbb{V}(\Gamma)$ by:

$$
\mathrm{cU}_{\mathrm{E}}(\mathrm{\Gamma}) \subseteq \mathrm{U}_{\mathrm{E}}(\mathrm{\Gamma})
$$$$
\forall \delta \in \mathrm{U}_{\mathrm{E}}(\Gamma) \quad \exists \sigma \in \mathrm{cU}_{\mathrm{E}}(\Gamma): \delta \geq_{\mathrm{E}} \sigma[\mathrm{W}]
$$

(correctness)

(completeness) 
A set of most general E-unifiers or minimal set of E-unifiers $\mu \mathrm{U}_{\mathrm{E}}(\mathrm{T})$ is a complete set with

$$
\forall \sigma, \tau \in \mu \mathrm{U}_{\mathrm{E}}(\Gamma): \sigma \geq_{\mathrm{E}} \tau[\mathrm{W}] \text { implies } \sigma=\tau \quad \text { (minimality). }
$$

If the set $\mu U_{E}(\Gamma)$ exists, it is unique up to the equivalence $\equiv_{E}$ [W] (see [Hu 76, FH 86]). Fages and Huet were the first who showed that $\mu U_{E}(\Gamma)$ need not exist in general. They gave a theory $E$ and terms s,t such that there is no minimal set of E-unifiers for $\langle s=t\rangle_{\mathrm{E}}$ [FH 86]. A more natural example is the theory AI (associativity and idempotence) of free bands (idempotent semigroups). Baader and Schrnidt-Schauß independently gave an AI-unification problem that has no minimal set of Al-unifiers [Ba 86, Sc 86a].

A possible reason for the non-existence of minimal sets of E-unifiers is that the corresponding quasi-ordering $\geq_{E}[W]$ on $U_{E}$ is not well-founded. Obviously, if the quasi-ordering is well-founded (every strictly decreasing chain in $U_{B}$ is finite), a minimal subset will always exist. More generally, if every decreasing chain of $E$-unifiers - also an infinite one - has a lower bound in $U_{E}$, then $U_{E}$ has a minimal subset (apply ZORN's Lemma to the set of $\equiv_{E}[W]$-equivalence classes of $U_{E}$ and the partial ordering induced by $\geq_{\mathrm{E}}[W]$ on this set). However, this condition is not necessary for the existence of minimal sets of E-unifiers: in Proposition 3.10 we present a theory $\mathrm{E}$, where every E-umification problem has a minimal set of $E$-unifiers, but some $E$-unifier sets contain infinite decreasing chains without a lower bound in the set of E-unifiers.

Usually the above definitions are carried out for unification problems that consist of only one equation, but we found an example presented in Theorem 3.9 that shows that this definition is not strong enough: there is a theory $E$ where all single term equations have minimal sets of E-unifiers, but for some equation systems minimal E-unifier sets do not exist. For that reason we define the following unification hierarchy for systems of equations. A theory $E$ is

$\begin{array}{lll}\text { - unitary unifying } & \text { iff } \mu \mathrm{U}_{\mathrm{E}}(\Gamma) \text { exists and }\left|\mu \mathrm{U}_{\mathrm{E}}(\Gamma)\right| \leq 1 \text { for all } \Gamma & \left(\mathrm{E} \in U_{1}\right) \\ \text { - finitary unifying } & \text { iff } \mu \mathrm{U}_{\mathrm{E}}(\Gamma) \text { exists and }\left|\mu \mathrm{U}_{\mathrm{E}}(\Gamma)\right|<\infty \text { for all } \Gamma & \left(\mathrm{E} \in \mathfrak{U}_{\omega}\right) \\ \text { - infinitary unifying } & \text { iff } \mu \mathrm{U}_{\mathrm{E}}(\Gamma) \text { exists for all } \Gamma \text { and }\left|\mu \mathrm{U}_{\mathrm{E}}(\Gamma)\right|=\infty \text { for some } \Gamma & \left(\mathrm{E} \in \mathfrak{U}_{\infty}\right) \\ \text { - nullary unifying } & \text { iff } \mu \mathrm{U}_{\mathrm{E}}(\Gamma) \text { does not exist for some } \Gamma & \left(\mathrm{E} \in U_{0}\right)\end{array}$

We use $U$ as an abbreviation for $U_{1} \cup \mathcal{U}_{\omega} \cup \mathcal{U}_{\infty}$

The proof of a result of Book and Siekmann [BS 84] implies (without any restrictions to the signature) that finitary theories are always unbounded: Given a finitary theory $E$, then for every $n \geq 1$ there exists an equation system $\Gamma$ with $\left|\mu \mathrm{U}_{\mathrm{E}}(\Gamma)\right|>\mathbf{n}$.

If we are only interested in substituting into the variables of one side (one-sided unification), we call such a problem an $E$-matching problem and write $\left\langle s_{\mathrm{i}} \gg \mathrm{t}_{\mathrm{i}}: 1 \leq \mathrm{i} \leq \mathrm{n}\right\rangle_{\mathrm{E}}$ for the problem to find a substitution $\sigma$ with $\operatorname{DOM}(\sigma) \subseteq \mathfrak{W V}\left(\mathrm{s}_{1}, \ldots \mathrm{s}_{\mathrm{n}}\right)$ such that $\mathrm{s}_{\mathrm{i}} \bar{F}_{\mathrm{E}} \sigma \mathrm{t}_{\mathrm{i}}(1 \leq \mathrm{i} \leq \mathrm{n})$. We say $\left(\mathrm{t}_{1}, \ldots, \mathrm{t}_{\mathrm{n}}\right)$ is E-matchable to $\left(\mathrm{s}_{1}, \ldots, \mathrm{s}_{\mathrm{n}}\right)$ and call $\sigma$ an E-matcher of $\left(t_{1}, \ldots, t_{n}\right)$ to $\left(s_{1}, \ldots, s_{n}\right)$. The set of all E-matchers of $\left(t_{1}, \ldots, t_{n}\right)$ to $\left(s_{1}, \ldots, s_{n}\right)$ is denoted by $\mathrm{M}_{\mathrm{E}}\left(\mathrm{s}_{\mathrm{i}}\right.$ " $\left.\mathrm{t}_{\mathrm{i}}: \mathrm{l} \leq \mathrm{i} \leq \mathrm{n}\right)$. Note that there is a difference between the matching relation and the instance relation since $f(x) \geq_{\varnothing} x$ but $M_{\varnothing}(f(x) \geqslant x)=\varnothing$ because $\mathbb{V}(x) \backslash V(f(x))=\varnothing$. Again we are interested in finding generating sets or a basis of matchers. In the same way as for unification we compare matchers by $\geq_{E}\left[V\left(\left(s_{i}, t_{i}\right): 1 \leq i \leq n\right]\right.$ and define complete and minimal set of E-matchers of $\left(t_{1}, \ldots, t_{n}\right)$ to $\left(s_{1}, \ldots, s_{n}\right)$ denoted by $\mathrm{cM}_{\mathrm{E}}\left(s_{\mathrm{i}} \gg \mathrm{t}_{\mathrm{i}}: 1 \leq \mathrm{i} \leq \mathrm{n}\right)$ and $\mu \mathrm{M}_{\mathrm{E}}\left(\mathrm{s}_{\mathrm{i}} \gg t_{\mathrm{i}}: 1 \leq \mathrm{i} \leq \mathrm{n}\right)$. Again minimal sets of matchers may not exist [FH 86]. Analogous to the unification hierarchy we can classify equational theories in unitary matching $\left(\mathrm{E} \in \mathcal{M}_{1}\right.$ ), finitary matching $\left(\mathrm{E} \in \mathscr{M}_{\omega}\right)$, infinitary matching ( $\mathrm{E} \in \mathscr{M}_{\infty}$ ) and nullary matching $\left(\mathrm{E} \in \mathscr{M}_{0}\right.$ ) theories. The class $\mathscr{M}_{1} \cup \mathscr{M}_{\infty} \cup \mathcal{M}_{\infty}$ is abreviated by $M$.

If we denote the set of substitutions $\sigma$ with $\operatorname{DOM}(\sigma) \subseteq M V\left(s_{1}, \ldots, s_{n}\right)$ by $\Sigma_{k N}(s, \ldots, s \mathrm{n})$, the set of all matchers is a left ideal in the monoid $\Sigma_{\mid n v(s 1, \ldots, s)}$, i.e. $M_{E}\left(s_{i} \gg t_{i}: 1 \leq i \leq n\right)=\Sigma_{\mid V v(s 1, \ldots, s n)} \cdot M_{E}\left(s_{j} \gg t_{i} ; 1 \leq i \leq n\right)$. An equivalent, and from a theoretical point of view more elegant definition of generating sets and bases of $E$-matchers can 
be achieved, if we define the instance relation only in this monoid $\Sigma_{\mid \mathrm{hv}\left(s 1_{1}, \ldots, 5\right)}$;

$$
\sigma Z_{E}^{*} \tau\left[W^{*}\right] \text { iff } \exists \lambda \in \Sigma_{\{W V(s 1, \ldots, s n)} \text { with } \sigma=_{E} \lambda \tau \text {, where } W^{*}=V\left(t_{1}, \ldots, t_{n}\right) \backslash V\left(s_{1}, \ldots, s_{n}\right) \text {. }
$$

Then completeness and minimality are defined with respect to the quasi-ordering ${ }^{*}{ }_{\mathrm{E}}\left[\mathrm{W}^{*}\right]$. This results in the same as considering the variables of the instance terms $s_{1}, \ldots, s_{n}$ as constants and performing E-unification.

Our definition is also equivalent to that of 'demi-unification' in [Hu 76] and to the matching definition in [Sz 82] (Szabó's restriction of admitting only idempotent matchers is not necessary, since it always can be satisfied).

In the literature there are more general definitions of matching, but they do not allow adequate definitions of minimal/complete sets of matchers to be representative. For example in the definition of completeness one may compare matchers only on $W=V\left(t_{1}, \ldots, t_{n}\right) V\left(s_{1}, \ldots, s_{n}\right)$ instead of $W=V\left(\left(s_{i j}, t_{j}\right): 1 \leq i \leq n\right)$ but then $c M_{E}\left(s_{i} \gg t_{i}: 1 \leq i \leq n\right)$ is not a generating set of the set of all matchers $M_{E}\left(s_{i} \gg t_{i}: 1 \leq i \leq n\right)$ : Consider the theory $E:=\{f(f(f(x)))=f(f(x))\}$ and the matching problem $\langle f(f(y)) » f(x)\rangle_{E}$ then there are two interesting matchers $\sigma=\{x \leftarrow f(y)\}$ and $\tau=\{x \leftarrow f(f(y))\}$ but both the matcher $\tau$ and the non-matching substitution $\tau^{\prime}=\{x \leftarrow f(f(a))\}$ are E-instances of $\sigma$ on $V(t) \backslash V(s)=\{x]: \tau \geq_{E} \sigma[\{x]]$ and $\tau^{\prime} \geq_{E} \sigma[[x]]$.

As mentionned above there is a difference between the matching relation and the instance relation. However, this restriction is not severe since in all practical applications of matching we have the additional hypothesis that the two terms are variable disjoint and in that case the two definitions are equivalent. On the other hand solving the 'instance relation problem' can be reduced to solving the matching problem, where all variables of the instance terms are renamed with new variables:

$$
\left\{\sigma \in \mathbb{E}: s_{i}=\sigma_{E} \sigma_{i}, 1 \leq i \leq n\right\}=\left\{\mu \rho: \mu \in M_{E}\left(s_{i} \gg \rho t_{i}: 1 \leq i \leq n\right)\right\},
$$

where $\rho$ is the renaming substitution $\left\{x \leftarrow v_{x}: x \in \mathbb{V}\left(s_{1}, \ldots, s_{n}\right)\right\}$ with pairwise different new variabies $\mathrm{v}_{\mathrm{x}} \in \mathbb{V} \backslash\left(\mathrm{s}_{\mathrm{i}}, \mathrm{t}_{\mathrm{i}}: \mathbf{1} \leq \mathrm{i} \leq \mathrm{n}\right)$.

Another way to overcome the difference between matching and instance relation would be to drop the restriction that matchers are in $\Sigma_{\left\{W_{(s 1, \ldots, s n)}\right.}$ and to use the matching definitions given in [FH 86]. But there we encounter analogous difficulties: If we compare the substitutions on $W=V\left(\left(s_{i}, t_{i}\right): 1 \leq i \leq n\right)$ then $\mu=\{x \leftarrow f(y)\}$ is not the only most general matcher of $\langle x \otimes f(y)\rangle_{\emptyset}$ since $\mu^{\prime}=\{x \leftarrow f(y), y \leftarrow z\}$ is a matcher but $\mu_{\emptyset} \mu^{\prime}[\{x, y\}]$. On the other hand, if we compare on $V\left(t_{1}, \ldots, t_{n}\right) V\left(s_{1}, \ldots, s_{n}\right)$ then a complete set $c M_{E}\left(s_{i} \gg t_{i}: 1 \leq i \leq n\right)$ does not generate the set of all matchers $M_{\mathrm{E}}\left(s_{\mathrm{i}} \gg \mathfrak{t}_{\mathrm{i}}: 1 \leq \mathrm{i} \leq \mathrm{n}\right.$ ) (use the same example as above).

\section{Some Classes of Equational Theories}

An equation $p=q$ with $\#(X, p)=\#(X, q)$ for every symbol $X \in \mathbb{V} \cup \mathbb{F}$ is calied a permutation equation. $A$ generalization of this notion is regularity. An equation $p=q$ is called regular iff $\mathbb{V}(p)=\mathbb{V}(q)$.

An equation is a collapse equation iff it is of the form $x=t$ where $t$ is a non-variable term and $x$ is a variable. Collapse equations of the form $p^{(i)}\left(v_{1}, \ldots, v_{i}, \ldots, v_{n}\right)=v_{i}$ (for some $i$ with $\left.1 \leq i \leq n\right)$ with pairwise different variables $\mathrm{v}_{1}, \ldots, \mathrm{v}_{\mathrm{n}}$ are called projection equations. An equation is called subterm collapsing iff one side of the equation is a proper subterm of the other. Of course in a consistent theory every collapse equation is subterm collapsing.

An equational theory $\mathrm{T}$ is called permutative iff every equation in $\mathrm{T}$ is a permutation equation, regular iff every equation in $\mathrm{T}$ is regular, and collapse free iff it does not contain any collapse equations. In [Ye 85] collapse free theories are called 'confined' and in [Ki 85] theories that contain collapse equations are said to be 'potent'. Examples for permutative and regular theories are associativity $A$ (presented by $f(x f(y z)=f(f(x, y) z)$ ) or commutativity $C$ (presented by $f(x y)=f(y x)$ ). The theory of idempotence $I$ (presented by $f(x, x)=x)$ is an example for a theory that is regular but not permutative and not collapse free. A theory $\mathrm{T}$ is almost collapse free iff for the leading function 
symbol of every collapse equation in $\mathrm{T}$ there is also a projection equation in $\mathrm{T}$. A theory $\mathrm{T}$ is called simple iff there is no subterm collapsing equation in T. Associativity and commutativity are examples for such theories.

An equational theory is said to be finite iff every equivalence class of the corresponding congruence is finite. An equational theory is Noetherian iff every strictly descending chain of substitutions is finite (i.e. in Noetherian theories the strict instance relations on substitutions are well-founded quasi-orderings). Another class of equational theories defined by the congruence ${ }_{E}$ is the class of $\Omega$-free theorics [Sz 82]. A theory is $\Omega-$ free iff $f\left(s_{1}, \ldots, s_{n}\right)=F_{E} f\left(t_{1}, \ldots, t_{n}\right)$ implies $s_{i}=_{B} t_{i}$ for all $1 \leq i \leq n$ and all function symbols $f \in \mathbb{F}$. Szabó called these theories $\Omega$-free since he denoted the signature by $\Omega$. A trivial example for an $\Omega$-free theory is the equational theory presented by the empty axiomatization $E=\varnothing$. Nontrivial examples are given below.

We study some problems in equational theories by transforming them into problems in monoids. This transformation yields a whole class of examples for equational theories, which are used in the following. A regular equational theory $\mathrm{T}$ is monadic iff all terms of some presentation are non-ground and built up with unary function symbols. For every monadic theory the set of non-ground monadic terms $\mathbb{T}_{1}=\mathbb{T}\left(F_{1},[x]\right)$ - terms built up with the variable $x$ and the unary function symbols $f \in \mathbb{F}_{1}$ - becomes a monoid with the unit element $x$ and the multiplication $s * t=\{x \leftarrow t\} s$. This monoid is isomorphic to the word monoid over the alphabet $F_{1}$ by the following transformation:

Every term $t=f_{1}\left(f_{2}\left(\ldots\left(f_{n}(x) \ldots\right)\right)\right.$ of $T_{1}$ is transformed into the $\mathbb{F}_{1}$-string $t_{M}=f_{1} f_{2} \ldots f_{n}$ and the variable $x$ is transformed into the empty word denoted by $x_{M}$.

With this transformation an axiomatization $E$ of a monadic theory induces a monoid $M_{E}$ with generating relations $s_{M}=t_{M}$ for $(s=t) \in E$, such that the following equivalence holds: $s={ }_{E} t \Leftrightarrow s_{M}{ }_{M} t_{M}$ *

Concerning unification theory the problem how to combine unification algorithms has been recently studied in a series of papers [He 86, Ki 85, Ti 86, Ye 85]. None of these approaches handles the class of collapse theories. A reason for the difficulty incorporating collapse theories into such algorithms is the fact that in collapse free theories the equivalence class of a variable only contains this variable, which is no longer true if we have collapse equations in the theory. One possiblity to get rid of some collapse equations is described in [Bü 86]. There it is shown that every almost collapse free theory can be transformed into a collapse free theory with the same unification behaviour.

In regular theories variables cannot disappear and all the terms of an equivalence class have the same variables. Another interesting property of those theories is that for every matching problem a minimal set of matchers always exists [Sz 82] (i.e. if $\mathrm{T}$ is a regular theory then $\mathrm{T} \in \mathcal{M}$ ).

Regularity, collapse freeness, and permutativity of an equational theory can be characterized by examination of an arbitrary presentation: it is sufficient to show that some presentation is regular, collapse free or permutative.

The definition of permutative theories is due to D.S. Lankford and A.M. Ballantyne [LB 77]. They introduced these theories in order to extend the Knuth-Bendix completion procedure to commutative theories. In recent papers as [Sz 82] or [Ki 85] permutative theories are defined as those theories that we call finite theories. We shall later give an example for a finite theory that is not permutative and we point out that the finiteness of the equivalence classes of an equational theory is undecidable. In finite theories the matching problems are always decidable and finitary matching [Sz 82]. Another important property of finite theories is that the set of most general unifiers always exists, i.e. there do not exist finite nullary unifying theories [Sz 82]. The reason for that fact is that finite theories are Noetherian. Obviously Noetherianness is sufficient for the existence of minimal sets of unifiers, but not necessary:

Proposition 3.1: Noetherian theories are in $u$, however the theory of idempotence $I$ is in $u$ but not Noetherian.

Note, that there exist theories that are finite (and hence Noetherian) and infinitary unifying (e.g. associativity) and that there exist theories that are finite (and hence Noetherian) and finitary unifying (e.g. commutativity). 
If we investigate unification problems then the class of simple theories plays a prominent role since they admit a simple occurs-sheck: a variable $x$ and a term $t$ are E-unifiable iff $x$ does not occur in $t$ Kirchner [Ki 85] combines unification algorithms by extending the method of Martelli and Montanari using multiequations [MM 82]. Given a set of multiequations a problem similar to the occurs-check has to be solved: It has to be checked whether there is a cycte in the multiequations or not. So Kirchner defines theories to be strict, iff every set of multiequations with a cycle is not E-unifiable [Ki 82].

Proposition 3.2: A theory $\mathrm{E}$ is simple iff $\mathrm{E}$ is strict.

Another useful property for the unification of multiequations is strong completeness of a theory $E$, i.e. every $E$-unifiable unification problem $\langle x=t\rangle_{E}$ has a complete set of E-unifiers, such that $\{x\}$ is the comain of every substitution in it $[\mathrm{Ki} 85]$. For instance the theory $E:=\{g(\mathrm{a} b)=a\}$ is not strongly complete since $\{x \leftarrow a, y \leftarrow b\}$ is a most general unifier of $\langle x=g(x y)\rangle_{E}$.

Proposition 3.3: Every simple theory is strongly complete.

The proof uses the following wellknown property: For a term $t$ and a variable $x$ with $x \notin \mathbb{V}(t)$ the substitution $(x \leftarrow 1\}$ is always a most general E-unifier.

An example for a non-simple, but strongly complete equational theory is the nullary unifying theory of associativity and idempotence [ $S \mathrm{c} 86 \mathrm{~b}$ ]. Beyond that if we want to apply certain results of Kirchner we have to show that a theory is strict and strongly complete. The last proposition shows that it is sufficient to show that the theory is simple.

Since finite theories are always simple (Lemma 3.6), the simple theories are also orthogonal to the unification hierarchy. In Theorem 3.4 we give an example of a simple nullary unifying theory.

The most important result about $\Omega$-free theories has been shown by Szabó: $\Omega$-free theories are regular and unitary matching and vice versa [Sz 82]. This result gives an algebraic characterization of a property which is defined in terms of unification theory. Ass it is shown later $\Omega$-freeness of an equational theory is undecidable, but on the other hand Szabó gave a sufficient condition for checking $\Omega$-freeness. Depending on the socalled groundcharacteristic set $\lambda_{0}(E)$ of a theory $\mathrm{E}$ - the union of all terms of the axiomatization and all their generalizations - he gives a criterion to check whether a theory is $\Omega$-free or not: a theory $E$ is $\Omega$-free iff all Robinson-unifiable terms $\mathrm{p}, \mathrm{q} \in \lambda_{0}(\mathrm{E})$ have exactly one most general $E$-unifier. Since $\lambda_{0}(E)$ is a finite set, this criterion yields a decision procedure for the $\Omega$-freeness of $E$, if a complete unification algorithm for $E$ is known. But also if such a unification algorithm is not known, one can nevertheless use this criterion to decide the $\Omega$-freeness of a given theory since sometimes it can be shown that a certain unification problem has at most one most general $E$-unifier. As proven in [Sz 82] $\Omega$-free theorics are again orthogonal to the unification hierarchy, i.e., there exist $\Omega$-free theories that are unitary unifying, finitary unifiying and infinitary unifying, respectively. We now give an example for a simple $\Omega$-free nullary unifying theory thus solving an open problem mentioned in [Sz 82], the existence of an $\Omega$-free theory of unification type nullary:

Theorem 3.4: There exists a simple, unitary matching (hence 2-free) theory of unification type nullary.

Sketch of Proof. Let $E$ be the monadic theory defined by the following canonical term rewriting system: $R_{E}:=\left\{f_{1}\left(g_{1}(x)\right) \rightarrow g_{2}\left(f_{1}(x)\right), f_{2}\left(g_{1}(x)\right) \rightarrow g_{2}\left(f_{2}(x)\right), f_{1}(k(x)) \rightarrow f_{2}(k(x)), g_{1}(k(l(x))) \rightarrow k(x)\right.$, $\left.g_{2}\left(f_{2}(k(l(x)))\right) \rightarrow f_{2}(k(x))\right)$. It can be shown by induction that this theory is simple and $\Omega$-free. The unification problem $\left\langle f_{1}(x)=f_{2}(x)\right\rangle_{E}$ has the complete set of unifiers: $\left\{\left(x \leftarrow g_{1}{ }^{n}(k(z))\right\} \mid n \geq 0\right\}$, but no most general unifier.

The $\Omega$-free theories are orthogonal to the other theories, i.e., there are examples of $\Omega$-free theories being permutative, finite, simple, collapse free, regular, respectively. Another feature of $\Omega$-free theories, is that every collapse axiom is 
built up only from unary function symbols [Sz 82]. Two series of examples for $\Omega$-free theories are given by the next lemma:

Lemma 3.5: (i) Every equational theory that is presented by a single ground axiom where both sides start with different function symbols is $\Omega$-free.

(ii) Let $\mathrm{T}$ be a monadic theory without free function symbols. Then the corresponding monoid $\mathrm{M}$ is a finitely presented group iff $\mathrm{T}$ is $\Omega$-free with a presentation consisting only of collapse axions.

So far we studied permutative, finite, simple, regular, collapse free, almost collapse free and $\Omega$-free theories. These classes are arranged in the following diagram in an inclusion hierarchy. We give examples for each possibility and counterexamples that show the inclusions are strict. For example the theory $E_{9}$ is simple and $\Omega$-free but not finite, hence it is regular, collapse free and almost collapse free. The inclusion proofs and the details showing that the particular theories listed above belong to the classes indicated in the diagram can be found in [BH 86].

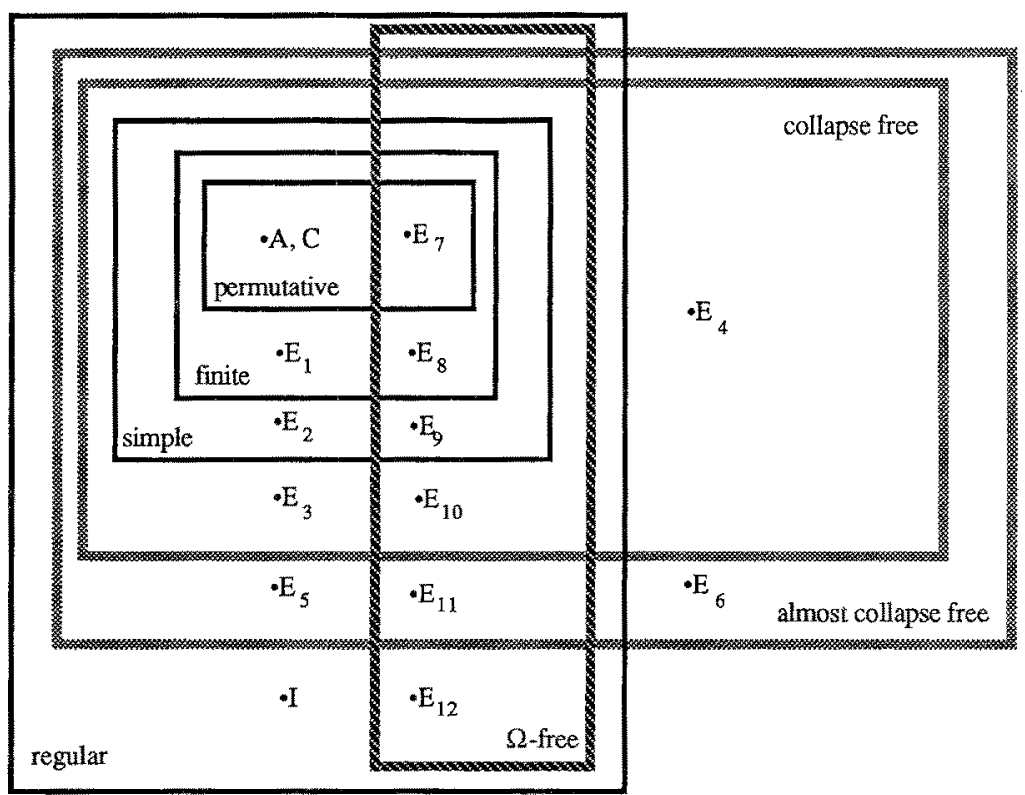

$$
\begin{aligned}
& A:=\{f(x f(y z))=f(f(x y) z)] \\
& C:=\{f(x y)=f(y x)\} \\
& I:=\{f(x x)=x\}
\end{aligned}
$$

$$
\begin{aligned}
& E_{1}:=\{f(a)=f(b)\} \\
& E_{2}:=\{f(g(x))=f(x)\} \\
& E_{3}:=\{f(x f(y y))=f(f(x x) y)\} \\
& E_{4}:=\{x * 0=0\} \\
& E_{5}:=\{f(a)=f(b), g(x)=x\} \\
& E_{6}:=\{g(x y)=x]
\end{aligned}
$$

$$
\begin{aligned}
& E_{7}:=\{f(g(a))=g(f(a))\} \\
& E_{8}:=\{f(a)=g(b)\} \\
& E_{9}:=\{f(g(h(x)))=g(x)\} \\
& E_{10}:=\{f(a)=a\} \\
& E_{11}:=\{f(g(x))=x, f(x)=x\} \\
& E_{12}:=\{f(g(x))=x, g(f(x))=x\}
\end{aligned}
$$

Noetherian theories are more interesting with respect to the unification hierarchy. Therefore we did not integrate this class in the hierarchy above. However, to get some intution for Noetherian theories we shall pick out some interesting relationships between the classes listed above and the Noetherian theories. 
Lemma 3.6: (i) Every finite theory is Noetherian.

(ii) There exists a finitary unifying theory that is not Noetherian.

(iii) There exists a Noetherian, but not regular theory.

Sketch of Proof. (ii) The theory $\mathrm{E}_{\mathrm{g}}=\{\mathrm{f}(\mathrm{g}(\mathrm{h}(\mathrm{x})))=\mathrm{g}(\mathrm{x})$ ) has a canonical term rewriting system. From [H] 80$]$ it follows that narrowing for this theory terminates, hence finite and minimal unifier sets exist for every term pair.

(iii) The theory $E:=[f(g(x))=a)$ is not regular but Noetherian.

Proposition 3.7: The theory $\Xi$ with the canonical term rewriting system

$$
R_{E}:=\left\{g_{1}\left(f_{1}\left(k\left(g_{3}(x)\right)\right)\right) \rightarrow f_{1}\left(h(x), g_{2}(k(x)) \rightarrow k\left(g_{3}(x)\right), h\left(f_{2}(x)\right) \rightarrow k\left(f_{2}(x)\right), h\left(g_{3}(x)\right) \rightarrow g_{2}(h(x))\right\}\right.
$$

is $\Omega$-free, simple and Noctherian but not finite.

Termination follows with a lexicographical ordering on terms. The theory is not finite, since we can reduce $g_{1}^{n+1}\left(f_{1}\left(k\left(g_{3}{ }^{n+1}\left(f_{2}(x)\right)\right)\right)\right)$ to $g_{1}^{n}\left(f_{1}\left(k\left(g_{3}{ }^{n}\left(f_{2}(x)\right)\right)\right)\right)$.

A simpler characterization of Noetherianness would be the requirement to have no infinitely descending chains of terms, but this does not hold in general. The theory $E:=\{g(h(x))=x, f(h(x))=f(x)\}$ has no infinitely descending chains of terms, but the theory is not Noetherian. However:

Lemma 3.8: If $E$ is $\Omega$-free, then $\Xi$ is Noetherian iff every descending chain of terms is finite.

We conclude this section with two interesting theories being counterexamples for certain intuitive assumptions in Inification theory; the first shows that there is a difference between unification of equation systems and unification of single equations as mentionned in Chapter 2 and the second shows that a theory is not necessarily mullary unifying if there is a unification problem with an infinite descending chain of unifiers without a lower bound in the set of unifiers.

Theorem 3.9: There exists a theory $E$ such that $\mu U_{E}(s=t)$ exists for all terms $s$ and $t$, however, $\mu \mathrm{U}_{\mathrm{E}}\left(\mathrm{s}_{1}=\mathrm{t}_{1}, \mathrm{~s}_{2}=\mathrm{t}_{2}\right)$ does not exist for some terms $\mathrm{s}_{1}, \mathrm{t}_{1}, \mathrm{~s}_{2}$ and $\mathrm{t}_{2}$.

Sketch of Proof. We give a regular, simple, $\Omega$-free and monadic theory that has the properties stated in the theorem. Let $E$ be the theory presented by the canonical term rewriting system with the following 11 rewrite rules:

$$
\begin{aligned}
R:= & \left(f_{i}\left(g_{1}(x)\right) \rightarrow g_{2}\left(f_{1}(x)\right), i=1,2,3,4\right. \\
& \left.f_{1}\left(k_{1}(x)\right) \rightarrow f_{2}\left(k_{1}(x)\right), f_{3}\left(k_{2}(x)\right) \rightarrow f_{4}\left(k_{2}(x)\right), k_{1}(h(x)) \rightarrow k_{2}(h(x)), g_{1}\left(k_{2}(h(l(x)))\right) \rightarrow k_{2} h(x)\right), \\
& \left.f_{1}\left(k_{2}(h(x))\right) \rightarrow f_{2}\left(k_{2}(h(x))\right), g_{2}\left(f_{2}\left(k_{2}(h(l(x)))\right)\right) \rightarrow f_{2}\left(k_{2}(h(x))\right), g_{2}\left(f_{4}\left(k_{2}(h(l(x)))\right)\right) \rightarrow f_{4}\left(k_{2}(h(x))\right)\right)
\end{aligned}
$$

The last three rules stem from the completion of the first eight rules. The equational theory has some symmetries: We can interchange i) $f_{1}$ and $f_{2}$, ii) $f_{3}$ and $f_{4}$, iii) $f_{1}, f_{2}, k_{1}$ and $f_{3}, f_{4}, k_{2}$ without changing the equational theory. The problem $\left\langle f_{1}(x)=f_{2}(x), f_{3}(x)=f_{4}(x)\right\rangle_{E}$ has a complete set $\left\{\left(x \leftarrow g_{1}{ }^{n}\left(k_{2}(h(z))\right)\right\}: n \geq 0\right\}$, but not a minimal one.

It is a pure technical task to construct theories $\mathrm{E}_{\mathrm{n}}$ from the above theory such that minimal sets of unifiers exist for all systems of n equations, but not for all systems of equations.

Proposition 3.10: There exists a theory $\mathrm{E} \in U$ such that there exists a unification problem $\Gamma$ and an infinite decreasing chain $\sigma_{1}>_{\mathrm{E}} \sigma_{2}>_{\mathrm{E}} \ldots$ of substitutions in $\mathrm{U}_{\mathrm{E}}(\Gamma)$ without a lower bound in $\mathrm{U}_{\mathrm{E}}(\Gamma)$.

Sketch of Proof: The theory $E$ presented by the canonical term rewriting system $R:=\left(f_{1}\left(g_{1}(x)\right) \rightarrow g_{2}\left(f_{1}(x)\right)\right.$, $f_{2}\left(g_{1}(x)\right) \rightarrow g_{2}\left(f_{2}(x)\right), f_{1}(k(x)) \rightarrow f_{2}(k(x)), g_{1}(k(h(l(x)))) \rightarrow k(h(x)), g_{2}\left(f_{2}(k(h(l(x)))) \rightarrow f_{2}(k(h(x)))\right)$ is regular simple and $\Omega$-free and the unification problem $\left\langle f_{1}(x)=f_{2}(x)\right\rangle_{E}$ has the property stated in the Proposition. 


\section{Decidability Results}

We finally investigate the decidability problems of the studied classes. The class problem of a class of equational theories is the problem whether a given equational theory belongs to this class. The undecidability of a class problem is guaranteed if it is shown for a subclass of this class. The uniform word problem for a class of equational theories is the problem to find an algorithm that decides the word problem for all equational theories. Permutativity, regularity and collapse freeness of a theory are easily decidable by examining a presentation of the theory.

Theorem 4.1 in [NO 85] shows that for finite Church-Rosser Semi-Thue-systems T the question "Does T admit any infinite congruence classes?" is undecidable. Hence the class problem for finite theories is undecidable.

The concept of a Markov property in monoids is a very useful tool for showing that certain properties of equational theories are undecidable. We show that some properties - for monadic theories - are Markov properties in the related monoid.

A property $P$ of finitely presented monoids is a Markov property, iff there exists a finitely presented monoid that has property $\mathrm{P}$ and one that has not property $\mathrm{P}$ and every monoid isomorphic to one with property $\mathrm{P}$ has property $\mathrm{P}$ itself. In [Ma 51, Mo 52] it is shown that such properties are undecidable.

Theorem 4.1: Almost collapse freeness, $\Omega$-freeness and Noetherianness of a theory are undecidable since these properties are Markov properties.

The simplicity of a theory is not a Markov property, since a simple theory turns into a non simple one by adding the axiom $\{f(x)=x\}$, but the corresponding monoid does not change. We show that the simplicity of an equational theory is undecidable by reducing it to the uniform word problem in simple equational theories.

Theorem 4.2: (i) The uniform word problem for ground terms in simple theories is undecidable.

(ii) The class problem for simple theories is undecidable.

Sketch of Proof. The first step is to show that for finitely generated, substitution invariant, regular equivalence relations on free terms it is undecidable whether two ground terms are in this relation. This is done by directly simulating a Turing machine, its lines correspond to one or more generating relations. By adding a new function symbol $h$ we get a simple theory $E$ with the axioms $h(s)=h(t)$ for every generating relation $s \sim t$, in which the word problem for ground terms is undecidable. The last step is to construct a theory $E^{\prime}:=E \cup\{s=a\} \cup\{t=f(a)\}$ with new symbols $\mathrm{f}$ and $\mathrm{a}$ and ground terms s,t. The decidability of simplicity of $\mathrm{E}^{t}$ would imply the decidability of $s={ }_{E} t$.

Finally it can be shown that for arbitrary theories it is undecidable where they reside in the unification hierarchy. By the asterisk in $\mathcal{U}_{i}^{*}$ and $\mathcal{M}_{i}^{*}$ we denote the interserction of $U_{i}$ and $M_{i}$ with the class of regular theories.

Theorem 4.3: It is undecidable whether an equational theory is in $u_{1}^{*}, u_{\omega}^{*}, U_{\infty}^{*}, u_{0}{ }^{*}, M_{1}^{*}, M_{\infty}{ }^{*}, M_{\infty}{ }^{*}$.

Sketch of Proof. The properties $\mathrm{E} \in \not_{i}{ }^{*}$ and $\mathrm{E} \in \mathcal{M}_{\mathrm{i}}^{*}$ for monadic $\mathrm{E}$ are invariant properties of the corresponding monoid, since they depend only on the congruence classes of $E$ and not on the presentation. In order to show that the above properties of theories are Markov properties it is sufficient to show that in every class there exists a monadic theory, For $u_{1}{ }^{*}$ and $M_{1}{ }^{*}$ choose the trivial monoid, for $u_{\omega}{ }^{*}$ and $\mathcal{M}_{\omega}{ }^{*}$ choose a finite monoid. For $u_{\infty}{ }^{*}$ and $M_{\infty}{ }^{*}$ the theory used in the proof of proposition 3.10 and for $u_{0} *$ the theory of proposition 3.9 are examples.

Corollary 4,4: The class problem for $u_{1}, u_{0}, u_{\infty}, u_{0}, \mathcal{M}_{1}, \mathcal{M}_{\omega}, \mathcal{M}_{\infty}$ is undecidable. 
Note that $\mathscr{M}_{0}$ is empty [Sz 82$]$ and the property $E \in M_{0}$ is not a Markov property. But we succeeded in reducing the undecidability of the class problem for $\mathcal{M}_{0}$ to the undecidability of the class problem for $u_{0}$.

Theorem 4.5: The class problem for $M_{0}$ is undecidable.

Sketch of Proof. Let $E$ be a monadic theory and let $\left.E^{\prime}:=f(x x)=a, f(x a)=a, f(a x)=a\right)$ such that $f$ and $a$ are not in $E$. Let $h$ be a (unary) function symbol not occurring elsewhere.

Let $E^{*}:=E \cup E^{\prime} \cup\{g(a)=a: g$ occurs in the axioms of $E\} \cup\{h(a)=a\}$. We assume that there is an infinite number of free constants available. Note that terms $s$ with $s \neq_{B}$ a behave as if the function symbols $f$ and $h$ are free. In a tedious proof it can be shown that the theory $\mathrm{E}^{*}$ is nullary matching iff $\mathrm{E}$ is nullary unifying. Now the result follows by the undecidability of the class problem for nullary unifying (monadic) theories.

Summarizing the results on the word problem in various ciasses, we conclude:

i) The uniform word problem in finite equational theories is decidable.

ii) The uniform word problem in simple theories is undecidable

iii) The uniform word problem for $\Omega$-free theories is undecidable.

The first holds, since in finite theories each equivalence class can effectively be generated. The second is proved above. The third follows from the undecidability of the word problem in groups using Lemma 3.5. It is open whether the word problem in Noetherian theories or finite and $\Omega$-free theories is decidable.

\section{Conclusions}

One aim of this paper was to unify the definitions of the classes of permutative, finite, simple, collapse free, almost collapse free, regular and $\Omega$-free equational theories. We studied the relationships between these theories and gave an inclusion hierarchy for them. We compared this hierarchy with the unification hierarchy and solved the decidability problems conceming these theories and the unification and matching hierarchy. Thereby we solved some of the open problems mentioned in the survey of Siekmann [Si 84]:

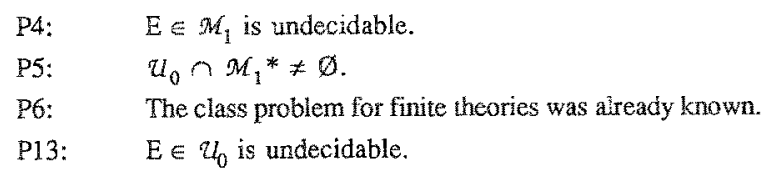

Finally we emphasize that when sudying unification in equational theories it is important not only to consider the axioms involved, but also the underlying term algebra, i.e., the set of function symbols involved. This accuracy resulted in the successful search for an example, for which the set of most general unifiers for a single equation always exists, but a system of equations bas no minimal set of unifiers. As a consequence we changed the definition of the unification hierarchy accordingly. 


\section{References:}

[Bi 35] Birkhoff, G.: On the Structure of Abstract Algebra, Proc. Cambrigde Phil. Soc., Vol. 31, p. 433-454, (1935)

[BH 86] Bürckert, H.-J., Herold, A. and Schmidt-SchauB, M.: On Equational Theories, Unification and Decidability, to appear as SEKI-Report SR-19-86, Universität Kaiserslautern, (1986)

[BS 81] Burris, S. and Sankappanavar, H.P.: A Course in Universal Algebra, Springer, (1981)

[BS 85] Book, R. and Siekmann, J.H.: On the Unification Hierarchy, Proc. of GWAI'85, Springer, p. 111-117, (1985)

[Bü 86] Bürckert, H.-J.: Some Relationships between Unification, Restricted Unification and Matching, in Proc. of the $8^{\text {th }}$ CADE, Springer, LNCS 230 , p. 514-524, (1986)

[FH 83] Fages, F. and Huet, G.: Unification and Matching in Equational Theories, Proc. of CAAP'83 (ed. G. Ausiello and M. Protasi), Springer, LNCS 159, p. 205-220, (1983). Also in TCS 43, pp. 189-200, (1986)

[GT 78] Goguen, J. A., Thatcher, J.W. and Wagner, E. G.: An Initial Algebra Approach to the Specification, Correctness and Implementation of Abstract Data Types, in Current Trends in Programming Methodology, Vol.4, Data Structuring (ed. R. T. Yeh), Prentice Hall, (1978)

[Gr 79] Grätzer, G.: Universal Algebra, Springer, (1979)

[He 86] Herold, A.: A Combination of Unification Algorithms, in Proc. of the $8^{\text {th }}$ CADE, Springer, LNCS $230, p$. 450-469, (1986)

[H1 80] Hullot, J.M.: Canonical Forms and Unification, in Proc. of $5^{\text {th }}$ CADE (eds. W. Bibel and R. Kowalski), Springer, LNCS 87, p. 318-334, (1989)

[HO 80] Huet, G. and Oppen, D. C.: Equations and Rewrite Rules: A Survey, in Formal Languages: Perspectives and Open Problems (ed R. Book), Academic Press, (1980)

[Hu 76] Huet, G.: Résolution d'équations dans des langages d'ordre 1,2 ... Université Paris VII, (1976)

[LB 77] Lankford, D.S. and Ballantyne, A.M.: Decision Procedures for Simple Equational Theories with Permutative Axioms: Complete Sets of Reductions, Report ATP-37, University of Texas, Austin, (1977)

[KB 70] Knuth, D.E. and Bendix, P.B.: Simple Word Problems in Universal Algebras, in Computational Problems in Abstract Algebras (ed. J. Leech), Pergamon Press, p. 263-297, (1970)

[Ki 85] Kirchner, C.: Methodes et outils de conception systematique d'algorithmes d'unification dans les théories équationelles, Thèse de doctorat d'état, (in French) Université de Nancy 1, (1985)

[Lo 78] Loveland, D.: Automated Theorem Proving: A Logical Basis, North-Holland, (1978)

[Ma 51] Markov, R.: Impossibility of Algorithms for Recognizing Some Properties of Associative Systems, Dokl. Akad. Nauk SSSR 77, p. 953-956, (1951).

[MM82] Martelli, A. and Montanari, U.: An Efficient Unification Algorithm, ACM TOPLAS, Vol.4, № 2, p. 258-282, (1982)

[Mo 52] Mostowski, A.: Review of [Ma 51], J. Symbolic Logic 17, p. 151-152, (1952)

[NO 85] Narendran, P., O'Dúnlaing, C. and Rolletschek, H.: Complexity of Certain Decision Problems About Congruential Languages, Journal of Computer and System Sciences, Vol.30, p. 343-358, (1985)

[Ro 65] Robinson, J. A.: A Machine-Oriented Logic Based on the Resolution Principle, JACM 12, №. 1, p. 23-41, (1965)

[Sc 86a] Schmidt-Schauß, M.: Unification under Associativity and Idempotence is of Type Nullary, Journal of Automated Reasoning 2,3, pp. 277-281, (1986)

[Sc 86b] Schmidt-Schauß, M.: Unification Properties of Idempotent Semigroups, SEKI Report SR-86-07, Universität Kaiserslautern, (1986)

[Si 84] Siekmann, J.: Universal Unification, in Proc. of $7^{\text {th }}$ CADE, Springer, LNCS 170, p. 1-42, (1984)

[Si 86] Siekmann, J.: Unification Theory, in Proc. of ECAI'86, Vol. II, p. vi-xxxv, Brighton, (1986)

[Sz 82] Szabó, P.: Unifikationstheorie Erster Ordnung, Dissertation (in German), Universität Karlsruhe, (1982)

[Ta 79] Taylor, W.: Equational Logic, Houston Journal of Mathematics 5, (1979)

[Ti 86] Tidén, E.: Unification in Combinations of Collapse-Free Theories with Disjoint Sets of Function Symbols, in Proc. of $8^{\text {th }}$ CADE, Springer, LCNS 230, p. 431-449, (1986)

[Ti 86b] Tidén, E.: First-Order Unification in Combinations of Equational Theories, Thesis, Stockholm, (1986)

[WR 67] Wos, L., Robinson, G.A., Carson, D. and Shalla, L.: The Concept of Demodulation in Theorem Proving, JACM 14, No. 4, p. 698-709, (1967)

[Ye 85] Yelick, K.: Combining Unification Algorithms for Confined Regular Equational Theories, in Proc. of RTA'85 (ed J.-P. Jouannaud), Springer, LNCS 202, p. 365-380, (1985) 